\title{
A114 ANTIBODIES AGAINST SYNDECAN-4 REDUCE CARTILAGE DESTRUCTION IN RA-LIKE DISEASE OF HTNF TRANSGENIC MICE
}

A Stratis, K Neugebauer, C Wehmeyer, C Wunrau, N Pundt, G Kollias, F Echtermeyer, T Pap Institute of Experimental Musculoskeletal Medicine, University Muenster, Muenster, Germany

\subsection{6/ard.2010.129635f}

Background Syndecan-4 is a transmembrane heparansulfate proteoglycan and one out of four members of the syndecan family described in mammals. Several studies have implicated syndecan- 4 in cell-matrix adhesion, cell migration, differentiation and proliferation, but its specific function in inflammatory pathologies remains unclear. Here, we used the human tumour necrosis factor $\alpha$ (TNF $\alpha$ ) transgenic (hTNFtg) mouse to analyse the expression and function of syndecan- 4 in chronic destructive arthritis and answer the question whether inhibition of syndecan- 4 by specific antibodies may prevent cartilage destruction in this animal model of human RA.

Methods Expression of syndecan- 4 was investigated by immunohistochemistry in the hind-paws of 8-week-old hTNFtg mice and wild type controls. In addition, synovial fibroblasts were isolated and analysed for syndecan- 4 expression by RT-PCR. For functional analyses, we generated blocking antibodies against syndecan-4. To investigate their effect on TNF $\alpha$ mediated destructive arthritis, hTNFtg mice were injected with the antibodies or with IgG control antibodies twice weekly for 4 weeks into their hind paws. Evaluation of disease severity included clinical parameters (weight, arthritis score, grip strength) as well as histomorphometric analysis of toluidin-blue stained paraffin sections and staining of disease-relevant MMPs

Results As seen in immunohistochemistry, there was a strong expression of syndecan- 4 in the synovial membranes of hTNFtg mice, whereas only negligible staining for syndecan- 4 was found in synovial tissues of wild type animals. In vitro, synovial fibroblasts isolated from hTNFtg mice showed more than 36.5-fold higher expression of syndecan-4 than wild type controls. Administration of the anti-syndecan- 4 antibodies but not of IgG control clearly ameliorated the clinical signs of arthritis $(p<0.05$ at week 8$)$ and protected the treated joints from cartilage damage. At histomorphometric analysis, this was evident for all analysed parameters but seen most prominently for area of distained cartilage (IgG control $23 \%$ vs anti-syndecan- $4 \%$, $p<0.05$ at week 8). Significantly reduced cartilage damage in the anti-syndecan- 4 treated hTNFtg mice was accompanied by a striking reduction in the expression of MMP3.

Conclusions Our findings indicate that syndecan- 4 is involved prominently in fibroblast-mediated cartilage damage in hTNFtg mice by regulating the expression of disease-relevant MMPs. More importantly, however, the data also suggest that inhibition of syndecan- 4 can prevent cartilage damage in this in vivo 


\section{EWRR abstracts}

model of human RA and that syndecan-4, therefore, may constitute a novel promising target for the treatment of this disease. 